\title{
Effects of Combination of Antidiabetic Agent and Statin on Alloxan-induced Diabetes with Cardiovascular Diseases in Rats
}

\author{
M. S. Islam ${ }^{1 \dagger}$, A. H. M. K. Alam ${ }^{1 \dagger}$, M. A. A. Rahman ${ }^{1}$, Y. Ali ${ }^{1}$, Al Mamun' ${ }^{2}$ M. Rahman ${ }^{3}$, \\ A. K. M. M. Hossain ${ }^{1}$, and M. Rashid ${ }^{1 *}$ \\ ${ }^{1}$ Department of Pharmacy, University of Rajshahi, Rajshahi-6205, Bangladesh \\ ${ }^{2}$ Department of Pharmacy, Jessore Science and Technology University, Jessore, Bangladesh \\ ${ }^{3}$ Department of Biochemistry and Molecular Biology, University of Rajshahi, Bangladesh
}

Received 25 February 2012, accepted in final revised form 1 June 2012

\begin{abstract}
The present study was designed to investigate the effects of combination drugs (metformin and atorvastatin) on long-term alloxan-induced diabetes with CVD in rats. In short-term alloxan-induced diabetic rats, metformin reduced significant amount of glucose in blood, but it had no significant effect on lipid profile. Atorvastatin significantly reduced TC, TG and LDL-C, whereas it increased significant amount of HDL-C. However, pathological changes of heart were not observed after short-term induction of alloxan in rats. In longterm induction of diabetes by alloxan, LV hypertrophy was observed and cardimyocyte size in rats was increased. Atorvastatin alone and in combination with metformin significantly reduced the LV hypertrophy, cardiomyocyte size, TC, TG and LDL-C level. They increased significant amount of HDL-C level and showed significant DPPH free radical scavenging activity. Present findings may suggest that treatment with combination therapy is more effective than mono-therapy for preventing diabetes with CVD in rats.
\end{abstract}

Keywords: Diabetes; CVD; Metformin; Atorvastatin; Combination drugs.

(c) 2012 JSR Publications. ISSN: 2070-0237 (Print); 2070-0245 (Online). All rights reserved. doi: http://dx.doi.org/10.3329/jsr.v4i3.9974 J. Sci. Res. 4 (3), 709-720 (2012)

\section{Introduction}

Diabetes is a major threat to global public health that is rapidly getting worse, and the biggest impact is on adults of working age in developing countries [1]. In most developing countries, at least one in ten deaths in adults aged 35 to 64 is attributable to diabetes, and in some the figure is as high as one in five [2]. Diabetes has become one of the major causes of premature illness and death in most countries, mainly through the increased risk of CVD (cardiovascular disease). In 2000, there were 171 million people with diabetes worldwide, and by 2030 this figure is expected to be more than double, to reach a total of 366 million [3]. Statistical projections for Bangladesh suggest that the number of diabetics will rise from 3.2 million in 2000 to 11.1 million in the year 2030 [4].

\footnotetext{
*Corresponding author: mamun69jp@yahoo.com
} 
Diabetes is usually accompanied by increased production of free radicals [5-7] or impaired antioxidant defenses [8], and this oxidative stress ultimately leads to apoptosis or myocardial cell injury and dysfunction by alteration of gene expression and modification of cellular responses [9]. Mechanisms by which increased oxidative stress is involved in the diabetic complications are partly known, including activation of transcription factors, advanced glycated end products and protein kinase C [6]. High glucose level also promotes intracellular lipid accumulation in vascular smooth muscle cells by impairing cholesterol influx and efflux balance [10]. Hypercholesterolemia and hypertriglyceridemia are independent risk factors that alone or together can accelerate the development of atherosclerosis and progression of atherosclerotic lesions [11-13].

At present, the treatment of diabetes mainly involves a sustained reduction in hyperglycemia by the use of drugs like biguanides, thiazolidinediones, sulfonylureas. However, due to unwanted side effects and a demand for the treatment of diabetes with CVD, the efficacies of these compounds are debatable [14, 15]. Moreover, long-term induction of diabetes without any lipid lowering drug leads to the risk of CVD. Hence, combination therapy has been suggested as a rich source of potentially useful antidiabetic and hypolipidemic drugs due to their ability to restore the function of pancreatic tissues by causing an increase insulin output, or to inhibit the intestinal absorption of glucose, or to facilitate the metabolites in insulin dependent processes [16], and their ability to inhibit the cholesterol biosynthetic pathway by inhibiting the enzyme HMG-CoA reductase [17]. Hence, treatment with combination therapy has an effect on protecting $\beta$-cells and smoothing out fluctuation in glucose level and cholesterol biosynthetic pathway. Therefore, the present study was aimed, at providing a strong view on experimental studies in animals, to find out the most effective and commonly used hypoglycemic and lipid lowering drugs combination on long-term alloxan-induced diabetes with CVD in rats.

\section{Materials and Methods}

\subsection{Animal studies}

All protocols for the animal experiments were reviewed and approved by the Animal Care and Use Committee of Institute of Biological Science, University of Rajshahi. LongEvans male rats weighing about 200-220 g, aged 2 months, were purchased from animal's house of International Centre for Diarrhoeal Disease Research, Bangladesh (ICDDRB). Prior to commencement of the experiments, all the rats were acclimatized to the new environmental condition for a period of one week. During the experimental period, the rats were kept in a well ventilated animal house at room temperature and were supplied standard pellets from ICDDRB and fresh drinking water. All the rats were kept in cages and maintained with natural 12 hour light and dark cycle. In both protocols (two weeks for short-term and four weeks for long-term), alloxan (120 mg/kg body weight, BW) was injected i.p. in rats. Alloxan-induced rats were treated with i.p. injection of metformin (500 mg/70 kg BW) and atorvastatin (20 mg/70 kg BW) for two weeks in the combination-therapy and for four weeks in the mono-therapy with metformin alone. 


\subsection{Measurement of glucose level}

Metformin, atorvastatin and combination of both were administered daily in alloxaninduced diabetic rats for both the protocols. After two and four weeks treatment with drugs, blood glucose level was determined two hours after last dose using glucometer (One Touch Horizon, USA).

\subsection{Measurement of lipid profile}

After completing the treatment of drugs, the rats were at first anesthetized with sodium phenobarbital. Then abdominal skin was cut and thoracic artery was opened. Finally 3-5 $\mathrm{ml}$ of blood was collected directly from thoracic artery by syringes. The blood was centrifuged at $4000 \mathrm{rpm}$ for 10 minutes and the serum was obtained. Serum lipid profile such as total cholesterol (TC), triglyceride (TG), low density lipoprotein-cholesterol (LDL-C) and high density lipoprotein-cholesterol (HDL-C), was assessed using diagnostic kits (Human, Germany).

\subsection{Evaluation of free radical scavenging activity}

The antioxidant activity using the 1,1-diphenyl-2-picrylhydrazyl (DPPH) was assessed as described previously [18]. The DPPH assay was performed by adding a sample (20 $\mu 1$ of blood plasma) plus $10 \mathrm{mM}$ sodium phosphate buffer ( $\mathrm{pH} \mathrm{7.4)}$ to $400 \mu \mathrm{l}$ of $0.1 \mathrm{mM}$ methanol solution of DPPH. After $30 \mathrm{~min}$ incubation at ambient temperature $\left(21^{\circ} \mathrm{C}\right)$, absorbance of the samples at $517 \mathrm{~nm}$ was measured. As precipitate was formed in most of the plasma samples, they were centrifuged before the measurement.

\subsection{Histopathological analysis}

Histological studies were performed for the determination of LV (Liver) weight and cardiomyocyte cross sectional area using previously reported procedure [19, 20]. In brief, $\mathrm{LV}$ weight was measured, and the ratio of LV weight to body weight was calculated to determine an index of LV hypertrophy. Four sections were obtained from each heart, and mounted on slides and stained with hematoxylin and eosin. To evaluate the extent of cardiomyocyte hypertrophy, cross-sectional images of cardiomyocyte were scanned at $x$ 400 magnifications. Approximately 20 cross-sections of cardiomyocyte were analyzed in each heart. Average values for each heart were used for analysis. All images were taken using an Olympus BX51TF (Olympus Corporation, Tokyo, Japan), and all measurements were determined using Scion Image software (Scion Corporation, Frederick, MD).

\subsection{Statistical analysis}

The results were expressed as mean \pm SEM. We used a one-way analysis of variance (ANOVA), followed by Dunnett's post-hoc test or students paired or unpaired $t$-test where appropriate. The statistical method applied in each analysis was described in each figure. Results were considered to be significant when $P$ values were less than $0.05(P<0.05)$. 


\section{Results}

The effects of drug alone (metformin/atorvastatin) and combination (metformin and atorvastatin) on the parameters of blood glucose level, lipid profile (TC, TG, LDL-C, HDL-C), antioxidant property and histopathology of heart (LV hypertrophy and cardiomyocyte size) were performed for both short and long-term alloxan-induced diabetes with CVD in rats.

\subsection{Effects of metformin and atorvastatin on blood glucose level in short-term alloxan- induced diabetic rats}

Short-term i.p. injection of alloxan in rats significantly increased blood glucose level (16 $\mathrm{mmol} / \mathrm{lit}$ ) when compared with normal rats $(5 \mathrm{mmol} / \mathrm{lit})$ (Fig. 1A). To clarify the individual effect of metformin and atorvastatin on blood glucose level, we estimated blood glucose level after two weeks treatment of metformin and atorvastatin on alloxaninduced diabetic rats. Metformin alone significantly decreased blood glucose level from $16.03 \pm 0.90$ to $5.837 \pm 0.66 \mathrm{mmol} / \mathrm{lit}$, whereas atorvastatin alone failed to reduce significant amount of blood glucose level when compared with alloxan-induced diabetic rats (Fig. 1A).

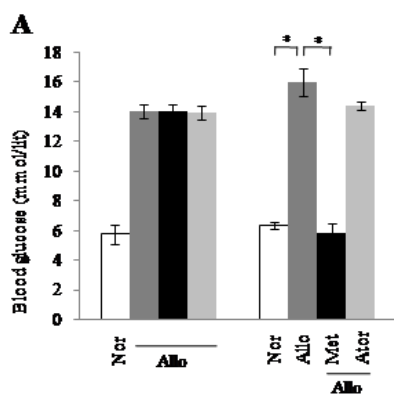

B

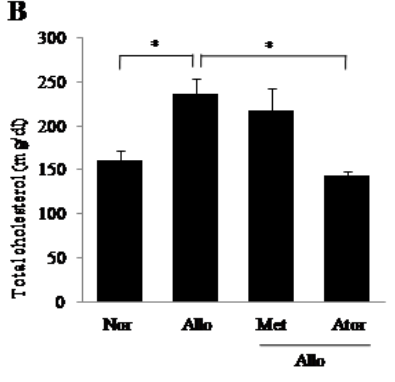

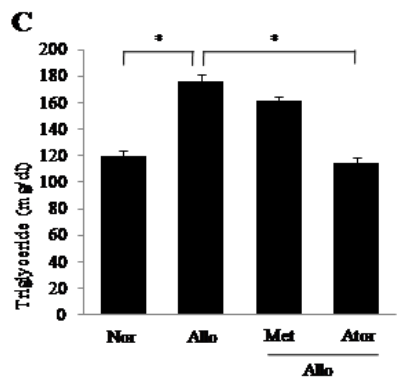
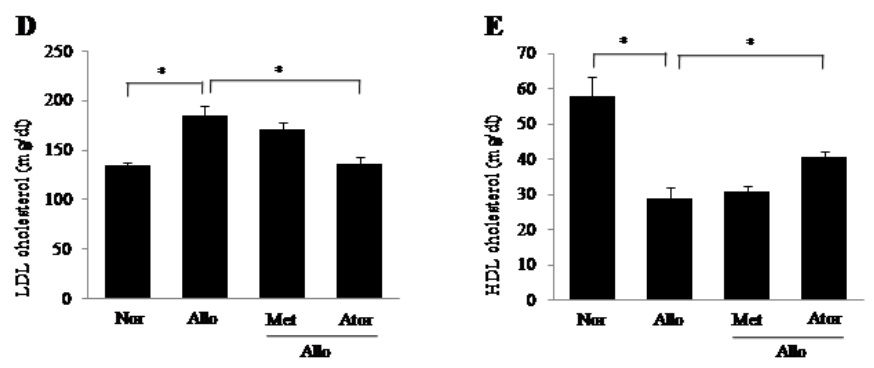

Fig. 1. Effects of metformin and atorvastatin for two weeks on blood glucose level and lipid profile in short-term alloxan-induced diabetic rats. (A) Blood glucose level; estimation of (B) TC, (C) TG, (D) LDL and (E) HDL-C levels. All values were presented as mean \pm SEM; $n=6$ in each group, ${ }^{*} P<0.05$ compared to alloxan-induced diabetic rats (ANOVA followed by Dunnett's test). Nor $=$ Normal, Allo $=$ Alloxan, Met $=$ Metformin, Ator $=$ Atorvastatin . 


\subsection{Effects of metformin and atorvastatin on lipid profile in short-term alloxan-induced diabetic rats}

Short-term induction of diabetes by alloxan in rats significantly altered lipid profile when compared with normal rats (Fig. 1B-E). To make clear the individual effect of metformin and atorvastatin on lipid profile, we examined TC, TG, LDL-C and HDL-C level after two weeks treatment of metformin and atorvastatin in diabetic rats. After two weeks treatment by drugs, we found that both metformin and atorvastatin reduced TC level $7.78 \%$ and 38.64\% (Fig. 1B), TG level $8.65 \%$ and 34.98\% (Fig. 1C), and LDL-C level $7.68 \%$ and 26.02\% (Fig. 1D), whereas they increased HDL-C level $6.95 \%$ and $41.73 \%$ (Fig. 1E), respectively when compared with diabetic rats. These results suggested that atorvastatin but not metformin acted as lipid lowering agents. We performed histological analysis in alloxan-induced rats and could not find any change of LV weight and cardiomyocyte size in diabetic rats.

\subsection{Effects of combination therapy on blood glucose level in long-term alloxan-induced diabetic rats}

Long-term induction of diabetes by alloxan in rats increased blood glucose level (32 $\mathrm{mmol} / \mathrm{lit})$ twice when compared with short-term induction of diabetes $(16 \mathrm{mmol} / \mathrm{lit})$ (Fig. $1 \mathrm{~A}$ and $2 \mathrm{~A})$. To investigate the effect of mono and combination therapy on blood glucose level in long-term alloxan-induced diabetic rats, we examined blood glucose level after four weeks treatment of metformin and atorvastatin alone and combination of both in diabetic rats. After four weeks treatment, metformin alone and combination of both significantly decreased blood glucose level from $28.54 \pm 2.19$ to $6.63 \pm 0.25 \mathrm{mmol} / \mathrm{lit}$ and $28.54 \pm 2.19$ to $5.38 \pm 0.56 \mathrm{mmol} / \mathrm{lit}$, respectively when compared with diabetic rats, but the effect of combination therapy on blood glucose level was higher than that of monotherapy alone. Atorvastatin failed to reduce significant amount of blood glucose level when compared with diabetic rats (Fig. 2A). Our results suggested that the combination therapy more effectively reduced the blood glucose level than mono-therapy in long-term alloxan-induced diabetic rats.

A

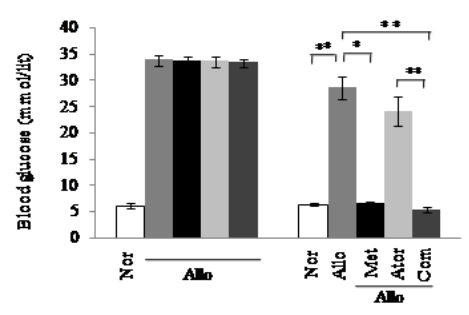

B

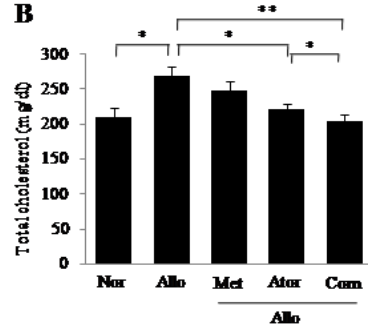

C

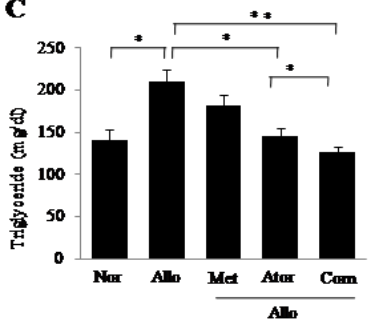



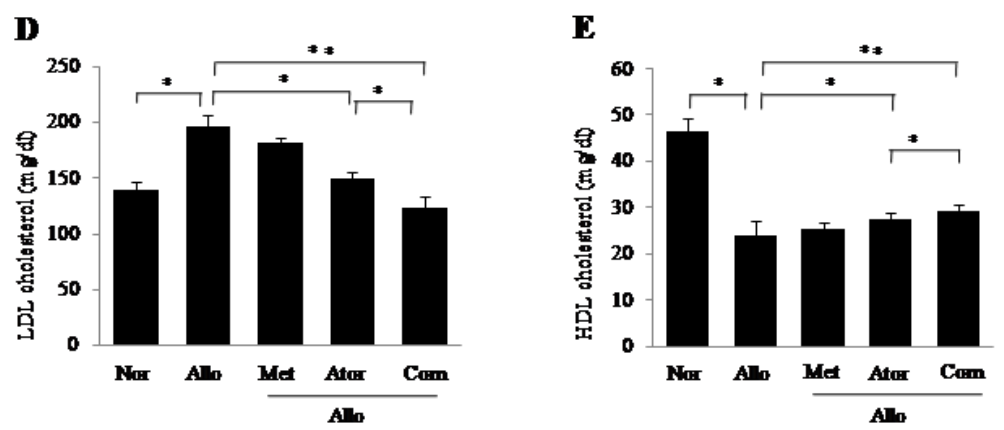

Fig. 2. Effects of metformin alone, atorvastatin alone and combination of both for four weeks on blood glucose level and lipid profile in long-term alloxan-induced diabetic rats. (A) Effect on blood glucose level; Estimation of (B) TC, (C) TG, (D) LDL and (E) HDL-C levels. Data were presented as mean $\pm \mathrm{SEM} ; n=6$ in each group, $* P<0.05$ and $* * P<0.01$ compared to alloxan-induced diabetic rats. Com $=$ combination (metformin and atorvastation).

\subsection{Effects of combination therapy on lipid profile in long-term alloxan-induced diabetic rats}

To clarify the effects of mono and combination therapy on lipid profile, we examined TC, TG, LDL-C and HDL-C level, after four weeks treatment with metformin and atorvastatin alone and combination of both in long-term alloxan-induced rats. After four weeks treatment, it was found that metformin, atorvastatin and combination of both reduced TC level 7.52\%, 17.65\% and 23.97\% (Fig. 2B), TG level 13\%, 30.4\% and 39.81\% (Fig. 2C), and LDL-C level $8.02 \%, 23.92 \%$ and 37.16\% (Fig. 2D), and they increased HDL-C level $5.20 \%, 14.58 \%$ and $21.87 \%$ (fig. $2 \mathrm{E}$ ), respectively when compared with diabetic rats. In contrast, the effect of combination therapy on lipid profile was higher than that of monotherapy alone. Metformin did not show significant effect on lipid profile (Fig. 2B-E). These results showed that combination therapy was more effective in lipid profile than the mono-therapy in long-term aloxan-induced diabetic rats.

\subsection{Effects of combination therapy on DPPH free radical scavenging activity on long- term alloxan-induced diabetic rats}

The effects of metformin, atorvastatin and combination of both on DPPH free radical scavenging activity were measured in long-term alloxan-induced diabetic rats. After four weeks treatment with metformin, atorvastatin and combination of both, it was observed that DPPH radical scavenging activity was increased 39.83\%, 65.65\% and $76.09 \%$, respectively when compared with normal control group (Fig. 3A). This result revealed that the combination therapy showed better antioxidant activity than mono-therapy in long-term alloxan-induced diabetic rats. 

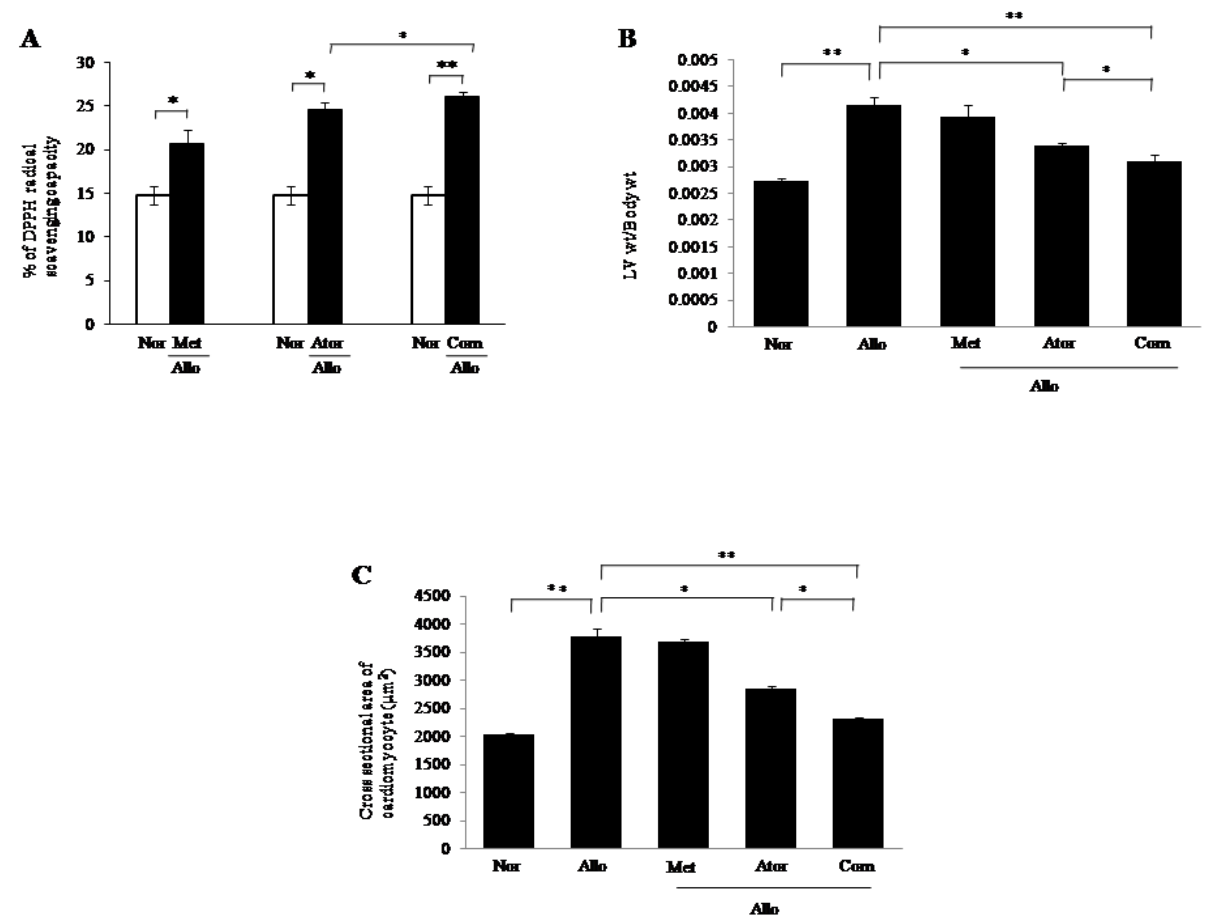

Fig. 3. Effects of metformin alone, atorvastatin alone and combination of both for four weeks on DPPH radical scavenging activity, LV hypertrophy and cardiomyocyte size in long-term alloxaninduced diabetic rats. (A) Percentage of DPPH radical scavenging activity; (B) Measurement of LV hypertrophy; (C) The effect on cardimyocyte size. The data were shown as mean \pm SEM, $n=6$ in each case. $* P<0.05$ and ${ }^{*} P<0.01$ compared to long term alloxan-induced diabetic rats (ANOVA followed by Dunnett's test).

\subsection{Effects of combination therapy on LV hypertrophy on long-term alloxan-induced diabetic rats}

Induction of diabetes by alloxan for four weeks in rats significantly increased LV hypertrophy (0.00416) when compared with normal rats (0.00274) (Fig. 3B). We examined the effect of single drug and combination of both on LV hypertrophy (LV weight/body weight) in long-term alloxn-induced diabetic rats to know whether or not the combination therapy was more effective than mono-therapy alone. After four weeks treatment, atorvastatin alone and combination therapy significantly reduced LV hypertrophy (0.00341) and (0.00310), respectively when compared with diabetic rats, and combination therapy reduced LV hypertrophy more effectively than mono-therapy (Fig. 3B). On the other hand, metformin alone did not significantly reduce LV hypertrophy (0.00395) when compared with diabetic rats. 


\subsection{Effects of combination therapy on cardiomyocyte size in long-term alloxan-induced diabetic rats}

Cardiomyocyte cross sectional area was significantly increased $\left(3775 \mu \mathrm{m}^{2}\right)$ in long-term alloxan-induced diabetes rats when compared with normal rats $\left(2041 \mu \mathrm{m}^{2}\right)$ (Fig. 3C). To make clear whether or not combination therapy was more effective than mono-therapy, we performed histopathological studies with four weeks treatment with metformin, atorvastatin and combination of both on diabetic rats. After four weeks treatment, atorvastatin alone and combination therapy significantly reduced cardiomyocyte cross sectional area $\left(2860 \mu \mathrm{m}^{2}\right)$ and $\left(2320 \mu \mathrm{m}^{2}\right)$, respectively when compared with diabetic rats (Fig. 3C). On the other hand, metformin did not significantly reduce cardiomyocyte cross sectional area $\left(3678 \mu \mathrm{m}^{2}\right)$. This result suggested that combination therapy was more effective in CVD than mono-therapy in long-term alloxan-induced diabetes rats.

\section{Discussion}

The risk of developing complications reduced substantially if diabetes can be effectively managed [21]. Diabetes, hyperlipidemia and CVD are a common debilitating illness among peoples in both developed and developing countries. Community surveys in industrialized countries have shown a prevalence of diabetes of $15-33 \%$ in people aged 30 years. The disease continues to be a leading cause of morbidity and mortality from the coronary artery disease and stroke. Simple life style adjustments, such as a healthy diet and physical activity, often combined with medication have been shown to be effective in promoting a full and healthy life with heart diseases. In many cases, type 2 diabetes mellitus accounting for over $90 \%$ of all cases of diabetes can be prevented through life style innervations alone. Though it may be true, but the maximum diabetic patient can not take care to prevent the diabetes through life style innervations. So, now a days, scientists and researchers all over the world are always engaged in research to find out new drug combination therapy and natural plant products to combat diabetes with CVD. The combination of metformin (antidiabetic drug) and atorvastatin (lipid lowering agent) may be used as an effective medicine in the treatment of diabetes and diabetes related risk factors especially CVD.

In the present study, diabetes was induced in rats by injecting alloxan (120 mg/kg BW) i.p. [22]. Alloxan is a cytotoxic agent induced diabetes in a wide variety of animal species by damaging insulin secreting $\beta$-cell, resulting in decrease of endogenous insulin release, which paved the way for the decrease utilization of glucose by the tissues. Hypercholesterolemia and hypertriglyceridemia are common complications of diabetes mellitus in addition to hyperglycemia [23]. This work has evaluated the effect of combination therapy of metformin and atorvastatin on diabetes in experimental animal of rats, and also on other important biochemical parameters, such as oxidative properties, LV hypertrophy and cardiomyocyte size which caused CVD after long-term induction of diabetes. 


\subsection{Effects of mono-therapy and combination therapy on blood glucose level}

The present study showed that metformin produced significant decrease in blood glucose level in alloxan-induced diabetic rats. On the other hand, atorvastatin did not produce any significant change of this parameter. Our findings supported the findings of previously published reports $[24,25]$. Interestingly, we observed that the combination therapy was more effective for controlling diabetes than the metformin alone in long-term alloxaninduced diabetic rats. However, further studies are needed to clarify the exact mechanism of this effect of combination therapy.

\subsection{Effects of monotherapy and combination therapy on lipid profile}

It was known that the factors which influence the glucose metabolism, under various physiological conditions, can influence lipid metabolism as well [26]. It had also been revealed that TG accumulation increased considerably in diabetes mellitus [27]. Hypercholesterolemia and hypertriglyceridemia had been reported to occur in diabetic [28]. A significant increase in TC, TG, and LDL-C level; and decrease in HDL-C level observed in our experiment as compared to normal rats in both protocol of diabetic rats. The serum TC, TG, LDL-C levels were significantly decreased and HDL-C level was significantly increased, after two weeks treatment with atorvastatin compared to alloxaninduced diabetic rats. We also found similar results on lipid profile after four weeks treatment with atorvastatin alone and combination therapy (metformin and atorvastatin) compared to alloxan-induced diabetic rats. Interestingly the combination therapy was more significant than atorvastatin alone. Metformin alone was lack of significance effect on lipid profile in both two and four week's studies. Our findings agreed with previously published reports [24, 25].

Recent evidences suggested that the serum lipids were usually increased in diabetes mellitus. For the distinct lipid lowering capacity of this combination therapy, it might be proposed that the drugs of the combination therapy may act as inhibitors for enzymes, such as hydroxyl-methyl-glutaryl-CoA reductase, which participates in de novo cholesterol biosynthesis.

\subsection{Effects of combination therapy on DPPH radical scavenging activity}

It had been reported that hyperglycemia increased oxidative stress, and oxidative stress was responsible for the CVD [6, 10]. Free radicals can cause damage to cardinal cellular components such as lipids, proteins, and nucleic acids (e.g. DNA), leading to subsequent cell death. The damage can become more widespread due to weakened cellular antioxidant defense systems. In our study, it was observed that treatment with metformin, atorvastatin and combination therapy significantly increased DPPH radical scavenging activity in long-term alloxan-induced diabetic rats. However, combination therapy showed higher antioxidant properties than single drug alone. It had been reported that statin 
showed pleiotrophic effect for reducing reactive oxygen species through inhibition of Rho/Rho-kinase and Rac1 signaling pathway [29, 30]. Our study may suggest that atorvastatin together with metformin might be more effective for reducing the oxidative stress associated with diabetes and CVD in rats.

\subsection{Effects of combination therapy on LV hypertrophy}

It had already been reported that long-term induction of diabetes without any treatment with cholesterol lowering agent produced CVD [31]. The present study revealed that longterm induction of alloxan produed LV hypertrophy in in vivo. The fact may be due to the increased production of oxidative stress by alloxan. Recent evident revealed that cardiovascular hypertrophy was associated with enhanced oxidative stress by upregulation of endothelial $\mathrm{NAD}(\mathrm{P}) \mathrm{H}$ oxidase and endothelial dysfunction [32]. Our present data showed that atorvastatin significantly reduced LV hypertrophy and combination of atorvastatin with metformin showed higher inhibitory effect on LV hypertrophy than mono-therapy.

\subsection{Effects of combination therapy on cardiomyocyte size}

Statin had a pliotrophic effect for reducing cardiovascular hypertrophy in vivo through inhibition of Rac1 signaling pathway [32]. The present study demonstrated that long-term induction of alloxan increased cardiomyocyte size in rats. Our present findings revealed that combination of atorvastatin with metformin was more effective than atorvastatin or metformin alone for ameliorating alloxan-induced increased cardiomyocyte size in vivo. These findings suggest the therapeutic potential of the combination of a statin and an antidiabetic drug for the treatment of cardiovascular hypertrophy.

\section{Conclusion}

Alloxan-induced diabetes in rats represents well-established animal model for both types of diabetes mellitus. Increased production of high levels of oxygen free radicals had been linked to glucose oxidation and non-enzymatic glycation of proteins. Furthermore, characteristic diabetes raised LDL-C level, lowered HDL-C level and elevated TG level which contributed the development of diabetic complications (such as CVD). Our study also revealed that long term induction of diabetes by alloxan produced CVD. The present study clearly indicated that the combination therapy possessed hypoglycemic, hypolipidemic effects and antioxidant property and had the beneficial effects for reducing cardiovascular hypertrophy (LV hypertrophy and cardiomyocyte size) in vivo. Therefore the present study suggested that atorvastatin together with metformin might be effective combination drug in the treatment of diabetes with CVD. However, further study is necessary to clarify the exact mechanism of inhibitory action of combination drugs on diabetes with CVD. 


\section{Acknowledgement}

The authors thank Square Pharmaceuticals Ltd, Bangladesh for its kind gift of atorvastatin and metformin. The authors also thank Professor Dr. Abdur Rashid, Faculty of Pharmacy, Department of Pharmaceutical Chemistry, University of Dhaka, for his generous gift of DPPH.

\section{References}

1. M. R. I. Khan, M. A. Islam, M. S. Hossain, M. Asadujjaman, M. I. I. Wahed, B. M. Rahman, A. S. M. Anisuzzaman, S. M. Shaheen, and M. Ahmed, J. Sci. Res. 2, 158 (2010). http://dx.doi.org/10.3329/jsr.v2i2.4020

2. S. Joan, J. Ame. Med. Ass. 29, 2534 (2004).

3. P. Rheeder, Fam. Pract. 48, 20 (2006).

4. S. Wild, G. Roghic, A. Green, R. Sinree, and H. King, Diabet Care 27, 1047 (2004). http://dx.doi.org/10.2337/diacare.27.5.1047

5. S. Pennathur, and J. W. Heinecke, Antioxid. Redox. Signal. 9, 955 (2007). http://dx.doi.org/10.1089/ars.2007.1595

6. H. A. Natheer, Diabet Vasc Dis Res. 8, 22 (2011). http://dx.doi.org/10.1177/1479164110390243

7. A. M. A. Amira, Res. J. Immunol. 3, 129 (2010). http://dx.doi.org/10.3923/rii.2010.129.145

8. F.E. Samson, and S. R. Nelson, Cell. Mol. Biol. 46, 699 (2000). PMid:10875433

9. S. Genet, R. K. Kale, and N. Z. Baquer, Mol. Cell. Biochem. 236, 7 (2002). http://dx.doi.org/10.1023/A:1016103131408

10. J. Xue, Z. Yuan, Y. Wu, Y. Liu, Y. Zhao, Y. Zhang, Y. Tian, W. Liu, Y. Liu, and C. Kishimoto, Cardio Res. 86, 141 (2010). http://dx.doi.org/10.1093/cvr/cvp388

11. J. M. McKenney, Cardio. Drugs Ther. 15, 413 (2001). http://dx.doi.org/10.1023/A:1013341606476

12. R. Yang, Y. Shi, G. Hao, W. Li, and G, Le, J. Clin. Biochem. Nutr. 43, 154 (2008). http://dx.doi.org/10.3164/jcbn.2008044

13. H. A. Ahad, B. S. Padmaja, P. Yesupadam, P. Guruprakash, M. Sravanthi and P. Ramyasree, J. Sci. Res. 3, 393 (2011). doi:10.3329/jsr.v3i2.7047

14. B. Noor, J. A. M. S. Rahman and K. Hossain, J. Sci. Res. 2, 67 (2010). DOI: $10.3329 /$ jsr.v2i1.2573

15. M. Asadujjaman, M. S. Hossain, M. R. I. Khan, A. S. M. Anisuzzaman, M. Ahmed, and A. Islam, Int. J. Phar. Sci. Res. 2, 1436 (2011).

16. N. Malviya, S. Jain, and S. Malvia, Acta. Pol. Pharma. Drug Res. 6, 7113 (2010).

17. P. Matafome, T. Louro, L. Rodriques, J. Crisostomo, E. Nunes, C. Amaral, P. Monterio, A. Cipriano, and R. Seica, Diabet Metab. Res. Rev. 27, 54 (2011). http://dx.doi.org/10.1002/dmrr.1157

18. A. Pieroni, S. Nebel, C. Quave, H. Munz, and M. Heinrich, J. Ethnopharmacol. 81, 165 (2002). http://dx.doi.org/10.1016/S0378-8741(02)00052-1

19. Y. Mukai, H. Shimokawa, T. Matoba, T. Kandabashi, S. Satoh, J. Hiroki, K. Kaibuchi, and A Takeshita, F. A. S. E. B. J. 15, 1062 (2001). PMid:11292668

20. M. Higashi, H. Shimokawa, T. Hattori, J. Hiroki, Y. Mukai, K. Morikawa, T. Ichiki, S. Takahashi, and A. Takeshita, Circ. Res. 93, 767 (2003). http://dx.doi.org/10.1161/01.RES.0000096650.91688.28

21. A. Kochhar, S. Dhindsa, and R. Sachdeva, Ethno-Med. 2, 107 (2008).

22. R. Vadivelan, P. Umasankar, M. Dipanjan, S. P. Dhanabal, A. Shanish, M. N. Satishkumar, and K. Elanko, Der. Pharma. Sinica. 2, 299 (2011). 
23. M. S. Hossain, M. Ahmed, and A. Islam, Int. J. Phar. Sci. Res. 1, 132 (2010).

24. N. Anitha, J. V. Rao, S. Kavimani, and V. Himabindu, J. Pharmaco. Toxicol. 3, 408 (2008).

25. R. Balasubramanian, S. Varadharajan, A. Kathale, L. M. Nagraj, I. Periyandavar, U. P. Nayak, A. Sharma, C. Bolmall, and V. P. Baliga, J. Ind. Med. Asso. 106, 464 (2008). PMid:18975505

26. D. J. A. Jenkins, A. L. Jenkins, T. M. S. Wolever, V. Vuksan, and A. V. Rao, Euro. J. Clin. Nut. 49, S68 (1995). PMid:8549564

27. S. G. Iams, and B. C. Wexler, Bri. J. Expt. Pathol. 58, 177 (1997).

28. S. R. Sharma, S. K. Dwivedi, and D. Swarup, Ind. J. Exp. Biol. 34, 372 (1996).

29. M. Takemoto, and J. K. Liao, Arterioscleros Thromb. Vasc. Biol. 21, 1712 (2001). http://dx.doi.org/10.1161/hq1101.098486

30. C. Maack, T. Kartes, H. Kilter, H. Schafers, G. Nickenig, M. Bohm, and U. Laufs, Circu. 108, 1567 (2003). http://dx.doi.org/10.1161/01.CIR.0000091084.46500.BB

31. H. Tsutsui, S. Kinugawa, S. Matsushima, and T. Yokota, J. Clin. Biochem. Nutr. 48, 68 (2011). http://dx.doi.org/10.3164/jcbn.11-012FR

32. M. Rashid, S. Tawara, Y. Fukumoto, M. Seto, K. Yano, and H. Shimokawa, Circu. J. 73, 361 (2009). http://dx.doi.org/10.1253/circj.CJ-08-0817 